\title{
The study of convex-dual-layer remote phosphor geometry in upgrading WLEDs color rendering index
}

\author{
Huu Phuc Dang', Nguyen Thi Phuong Loan², Nguyen Thi Kim Chung ${ }^{3}$, Nguyen Doan Quoc Anh \\ ${ }^{1}$ Faculty of Fundamental Science, Industrial University of Ho Chi Minh City, Vietnam \\ ${ }^{2}$ Faculty of Fundamental 2, Posts and Telecommunications Institute of Technology, Vietnam \\ ${ }^{3}$ Thu Dau Mot University, Thu Dau Mot City, Binh Duong Province, Vietnam \\ ${ }^{4}$ Power System Optimization Research Group, Faculty of Electrical and Electronics Engineering, Ton Duc Thang \\ University, Ho Chi Minh City, Vietnam
}

\section{Article Info}

Article history:

Received Sep 8, 2020

Revised Apr 19, 2021

Accepted Apr 30, 2021

\section{Keywords:}

$\mathrm{CaGa}_{2} \mathrm{~S}_{4}: \mathrm{Mn}^{2+}$

Dual-layer remote

Mie-scattering theory color

rendering index

Phosphor geometry

WLEDs

\begin{abstract}
The white-light light-emitting diode (LED) is a semiconductor light source that usually has one chip and one phosphor layer. Because of that simple structure, the color rendering index (CRI) is really poor. Therefore, structure with double layer of phosphor and multiple chips has been studied with the phosphorus proportions and densities in the silicone are constantly changed to find the best option to improve optical properties. In research, we use red phosphor Ca5B2SiO10:Eu3+ layer to place above the yellow phosphor one, and both of them have a convex design. Then, the experiments and measurements are carried out to figure out the effects of this red phosphor as well as the convex-double-layer remote phosphor design on the LED's performances. The measured results reveal that the light output is enhanced significantly when using convex-dual-layer structure instead of the singlelayer design. Additionally, the $\mathrm{Ca} 5 \mathrm{~B} 2 \mathrm{SiO} 10: \mathrm{Eu} 3+$ concentration benefits CRI and CQS at around $6600 \mathrm{~K}$ and $7700 \mathrm{~K}$ correlated color temperature (CCT). Yet, the lumen output shows a slight decline as this red phosphor concentration surpass $26 \%$ wt. Through the experiments, it is found that a double layer of chip and double phosphorus is the best structure which could support the quality of CRI and luminous flux.
\end{abstract}

This is an open access article under the CC BY-SA license.

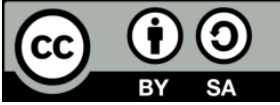

\section{Corresponding Author:}

Nguyen Doan Quoc Anh

Faculty of Electrical and Electronics Engineering

Ton Duc Thang University,

No. 19 Nguyen Huu Tho Street, Tan Phong Ward, District 7, Ho Chi Minh City, Vietnam

Email: nguyendoanquocanh@tdtu.edu.vn

\section{INTRODUCTION}

Over the past few years, the demand of high-efficient lighting source has been increasing. There are some requirements that a qualified lighting source must meet to be applied in advanced illuminating applications such as high durability, power saving, robustness, waste-free, and small size. The white-light light-emitting diode (LED) is an extremely potential option that fully meets those conditions [1]. With the fast-switching advancement, LEDs got more and more attention for manufacturers and have been applied in many state-of-the-art technologies, including smart lighting and auto lighting. Besides, in the aspect of biomedical imaging, LEDs were noticed as a light source for high-resolution full-field optical coherence microscopy (FF-OCM) [2], [3]. However, due to the low illuminating performance, their applications have been restricted in many fields, which probably is the main force for researchers and manufacturers to improve the lighting performances of WLEDs. To assess the lighting performance of the LED we need to 
rely on the color rendering index (CRI) to compare the light radiation from LED to a natural light source, such as sunlight, or black-body radiation. If the phosphor's emission spectrum in the LED matches the blackbody radiation's broad continuous spectrum, the CRI is high and so is the light quality [4]-[6].

There are many ways to construct a white-light emitting structure with LED chips and phosphor; however, the configuration containing one blue chip and yellow phosphor is the most common because of cheap production cost [7], [8]. Although this structure is most commonly used, the constant usage gradually revealed that this structure is not optimal for lighting efficiency. This is concluded after considering the low CRI results despite many enhancing efforts and high deterioration rate of this structure. According to recent researches, the color rendering index can reach higher values when adjusting the emission spectra to desired value by combining appropriate phosphor materials and blue LED chips [9]-[12]. In order to correct the issue with light color and CRI, we conducted a dissection of LED and phosphor spectrum with empirical and mathematical computations for more information. We have also known that the biggest obstacle for customized white LEDs having adjustable correlated color temperatures (CCTs) is to achieve high CRI and maintain high lumen efficacy simultaneously. The package with one blue LED chip and two layers of phosphor can result in good CRI but show low lighting performance because of Stokes shift [13]-[15]. On the other hand, the package of blue and red LED chips combined with a phosphor film can help both CRI and efficacy but is unable to adjust the color like the two phosphors package [16]-[19]. Thus, employing more LED chips and phosphor materials seems to be the solution for advancing WLED CRI, lumen efficiency and color tunability. Therefore, we decided to use 9 LED chips and two phosphors to fabricate the WLED configuration and analyzed the effects of the phosphors on the optical properties of the WLED.

In this study, the color design based on the Beer-Lambert law and linear conversion was investigated in relation to the fabrication demands of subtle chromatic discrepancy for white-light LEDs. The white-light LED packages in the experiments are comprised of yttrium aluminum garnet (YAG), blue chips, and a layer of red phosphor Ca5B2SiO10:Eu3+. The different phosphor proportions and densities are used to create a dual-layer remote phosphor structure with convex shape and to conduct a thorough analysis of the performance of LEDs. The results show that the proposed convex-dual-layer design is easy to fabricate and yields high efficiency. Furthermore, the information this study provided is crucial to the further development of WLEDs, especially when high-illuminating WLEDs are seen as a potential for being applied in imaging techniques such as optical coherence tomography (OCT) [20].

\section{PREPARATION AND SIMULATION}

\subsection{Preparation}

The first step to prepare for a successful experiment is to understand the nature of $\mathrm{Ca}_{5} \mathrm{~B}_{2} \mathrm{SiO}_{10}: \mathrm{Eu}^{3+}$ through its ingredients: $100 \mathrm{~g}$ of $\mathrm{CaCO}_{3}$ (with a very large majority of $\mathrm{Ca}$ ), 8.8 gram of $\mathrm{Eu}_{2} \mathrm{O}_{3}$ (with one in twenty of $\mathrm{Eu}$ ), $13.2 \mathrm{~g}$ of $\mathrm{SiO}_{2}$ (with one in fifth of $\mathrm{Si}$ ) and $31 \mathrm{~g}$ of $\mathrm{H}_{3} \mathrm{BO}_{3}$. Secondly, it is essential to accurately execute the process of creating $\mathrm{Ca}_{5} \mathrm{~B}_{2} \mathrm{SiO}_{10}: \mathrm{Eu}^{3+}$. The first step is to mix the substances by dry milling or grinding. Next, fire the combination in open quartz boats in the air and at a temperature of 1100 degrees Celsius for 1 hour; after milling, we will get the mixture powderized. Moving on to the second step, let's take the previous combination and fire in the same condition but at 1200 degrees Celsius. Afterwards, we will also powderize the result of the second process. Finally, fire them again at 1300 degrees Celsius. The fired product is powderized for the last time and stored. The process of creating $\mathrm{Ca}_{5} \mathrm{~B}_{2} \mathrm{SiO}_{10}: \mathrm{Eu}^{3+}$ is seen as successful when the combination can acquire the following optical properties. During the emission, the compound has a red color. In addition, its emission peaks belong to $2.03 \mathrm{eV}$. Moreover, - (4.88 $\mathrm{eV})$ and $-(3.40 \mathrm{eV})$ are the indices of excitation efficiency by $\mathrm{UV}$.

\subsection{Simulation}

In this report, both the LightTools program and the Mie-theory are utilized to create the simulation of the LED and for experiments and results verification [21], [22]. After successfully fabricating the red phosphor $\mathrm{Ca}_{5} \mathrm{~B}_{2} \mathrm{SiO}_{10}: \mathrm{Eu}^{3+}$, the WLED dual-layer remote phosphor structure can be simulated with the correlated color temperature of $5600 \mathrm{~K}$. Then, the experimented can be carried out to investigate the influences of this red phosphor on the lighting performances. Figure 1 demonstrates how the $\mathrm{Ca}_{5} \mathrm{~B}_{2} \mathrm{SiO}_{10}: \mathrm{Eu}^{3+}$ and YAG:Ce ${ }^{3+}$ phosphor layers are organized in the in-cup phosphor configuration of WLEDs. As can be seen, the model in Figure 1 includes blue chips, a reflector cup, phosphor layers, and a silicone layer. The order of the phosphor layers is $\mathrm{Ca}_{5} \mathrm{~B}_{2} \mathrm{SiO}_{10}: \mathrm{Eu}^{3+}$ phosphor, the yellow YAG: $\mathrm{Ce}^{3+}$ phosphor, and the silicone. The reflector here is used to cover the LED chips to prevent them from environmental impacts. The size of this reflector is $2.07 \times 8 \times 9.85 \mathrm{~mm}$ in depth $\mathrm{x}$ bottom length $\mathrm{x}$ top surface length. Each blue chip has an emission power of $1.16 \mathrm{~W}$ at the peak wavelength of $453 \mathrm{~nm}$. The refractive index of $\mathrm{Ca}_{5} \mathrm{~B}_{2} \mathrm{SiO}_{10}: \mathrm{Eu}^{3+}$ phosphor is 1.85 and $\mathrm{YAG}: \mathrm{Ce}^{3+}$ is 1.83. The concentration of $\mathrm{YAG}: \mathrm{Ce}^{3+}$ needed to be adjusted according to $\mathrm{Ca}_{5} \mathrm{~B}_{2} \mathrm{SiO}_{10}: \mathrm{Eu}^{3+}$ concentration to keep the average CCTs. 


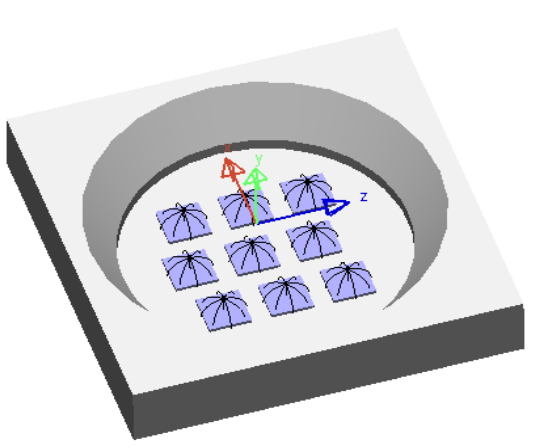

(a)

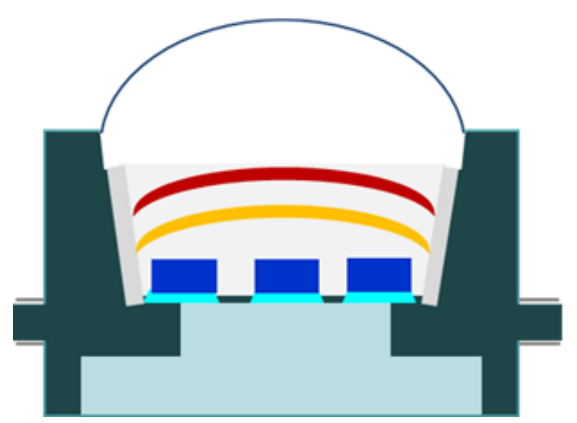

(c)

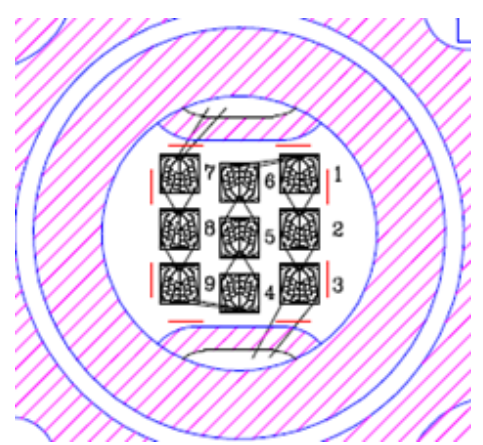

(b)

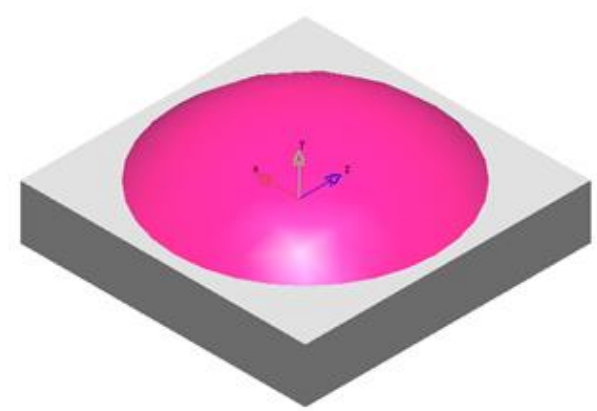

(d)

Figure 1. How the Ca5B2SiO10:Eu3+ and YAG:Ce3+ phosphor layers are organized in the in-cup phosphor configuration of WLEDs; (a) 3D modelling, (b) bonding diagram, (c) model of pc-WLEDs cross-section, (d) illustration of WLEDs simulated from LightTools commercial software

\section{RESULTS AND DISCUSSION}

The variations of YAG:Ce ${ }^{3+}$ phosphor to keep the average CCTs when $\mathrm{Ca}_{5} \mathrm{~B}_{2} \mathrm{SiO}_{10}: \mathrm{Eu}^{3+}$ phosphor increases are shown in Figure 2. In particular, with the concentration of $\mathrm{Ca}_{5} \mathrm{~B}_{2} \mathrm{SiO}_{10}$ : $\mathrm{Eu}^{3+}$ rising from $2 \%$ to 26\% wt., the concentration of YAG: $\mathrm{Ce}^{3+}$ phosphor needs to be decreased to keep the average CCTs. The variation based on these results also affects scattering and absorption properties, thus, it can lead to potential improvements in WLEDs color quality and light output. Therefore, choosing the right concentration of $\mathrm{Ca}_{5} \mathrm{~B}_{2} \mathrm{SiO}_{10}: \mathrm{Eu}^{3+}$ is a crucial requirement to ensure the performance of phosphor in WLEDs.

In Figure 3, the effects of red phosphor $\mathrm{Ca}_{5} \mathrm{~B}_{2} \mathrm{SiO}_{10}: \mathrm{Eu}^{3+}$ in the concentration range of $2 \%-24 \%$ are described on emission spectra of WLEDs. As can be seen, there are three spectral regions at which the growth in red-light components was observed when the $\mathrm{Ca}_{5} \mathrm{~B}_{2} \mathrm{SiO}_{10}: \mathrm{Eu}^{3+}$ concentration increases. In particular, the most notable spectral region is $648-738 \mathrm{~nm}$, and the other two are $420-480 \mathrm{~nm}$ and $500-640 \mathrm{~nm}$. The blue light falls into the $420-480 \mathrm{~nm}$ of the emission spectrum, thus, also benefits from the spectrum enhancement and achieve better quality. Additionally, the higher color temperature resulted in greater emission spectra. The results confirm that the presence of $\mathrm{Ca}_{5} \mathrm{~B}_{2} \mathrm{SiO}_{10}: \mathrm{Eu}^{3+}$ at low color temperature $(6600 \mathrm{~K})$ and high color temperature $(7700 \mathrm{~K})$ will be very helpful for the color quality of WLEDs. This is a crucial finding that influences the selection of the correct concentration to manufacture an optimal LEDs device. Yet, the reduction luminous efficiency is also noticed when the color quality of the WLED is improved. Figure 4 demonstrated the benefits of using $\mathrm{Ca}_{5} \mathrm{~B}_{2} \mathrm{SiO}_{10}: \mathrm{Eu}^{3+}$ red phosphor in improving the color rendering index (CRI) of WLED. This enhancement can be attributed to $\mathrm{Ca}_{5} \mathrm{~B}_{2} \mathrm{SiO}_{10}: \mathrm{Eu}^{3+}$ absorption feature. Particularly, the red phosphor $\mathrm{Ca}_{5} \mathrm{~B}_{2} \mathrm{SiO}_{10}: \mathrm{Eu}^{3+}$ also absorbs yellow lights but the blue-light absorbance is more dominant. As a result, the red light components in WLEDs increase when the package contains the phosphor $\mathrm{Ca}_{5} \mathrm{~B}_{2} \mathrm{SiO}_{10}: \mathrm{Eu}^{3+}$, which is beneficial to the CRI. Color rendering index is one of the valuable indicators that can evaluate the quality of WLEDs. The WLEDs with high CRI are considered to be higher quality; however, it also leads to higher production cost. Nevertheless, utilizing $\mathrm{Ca}_{5} \mathrm{~B}_{2} \mathrm{SiO}_{10}: \mathrm{Eu}^{3+}$ phosphor can lower the cost; thus, this material turns out to be one of the suitable candidates for large-scale production of WLEDs with higher color quality.

In recent years, CRI is not the only index to color quality of WLEDs as it is not a very in-depth indicator. Instead, the color quality scale (CQS) has been investigated and considered as a superior to CRI because CQS can examine not only CRI but also the viewer's preference and color coordinates. Figure 5 
shows the influences of the red phosphor $\mathrm{Ca}_{5} \mathrm{~B}_{2} \mathrm{SiO}_{10}: \mathrm{Eu}^{3+}$ on the CQS that helps enhance the quality of color. The ability of enhancing color quality for WLEDs of $\mathrm{Ca}_{5} \mathrm{~B}_{2} \mathrm{SiO}_{10}: \mathrm{Eu}^{3+}$ phosphor has been verified yet there is a drawback that should be considered: the reduction in the amount of emitted light. The mathematical formulas for computing the transmitted blue light and the converted yellow light in the convex-dual-layer remote phosphor structure, a potential part that can enhance the efficiency of LEDs, are presented in the next part.

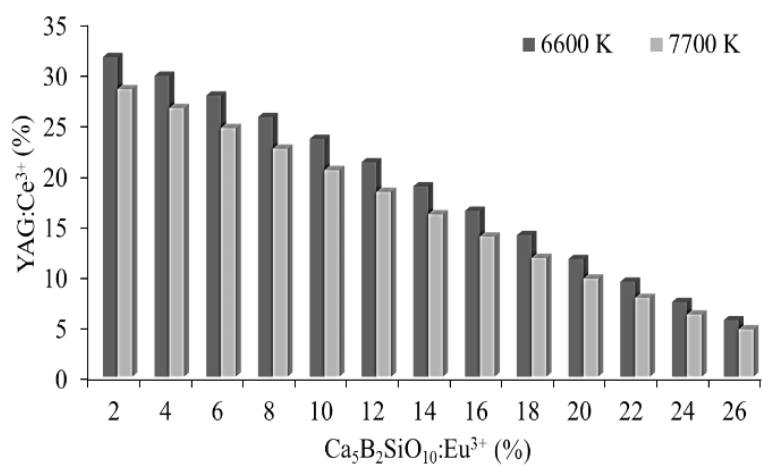

Figure 2. The change in YAG: $\mathrm{Ce}^{3+}$ concentration as a function of $\mathrm{Ca}_{5} \mathrm{~B}_{2} \mathrm{SiO}_{10}: \mathrm{Eu}^{3+}$ content

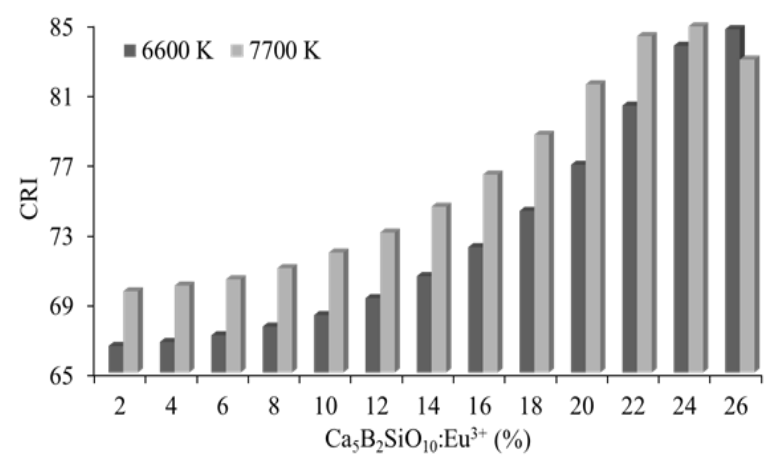

Figure 4. The color rendering index of WLEDs corresponding to $\mathrm{Ca}_{5} \mathrm{~B}_{2} \mathrm{SiO}_{10}: \mathrm{Eu}^{3+}$ concentration

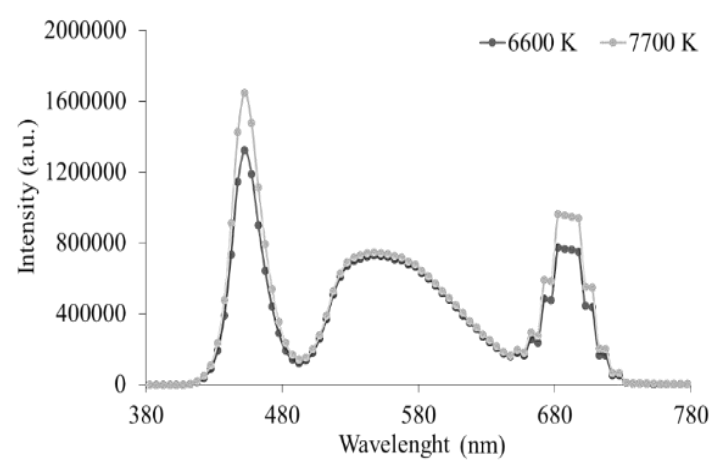

Figure 3. The emission spectra of WLEDs corresponding to $\mathrm{Ca}_{5} \mathrm{~B}_{2} \mathrm{SiO}_{10}: \mathrm{Eu}^{3+}$ concentration

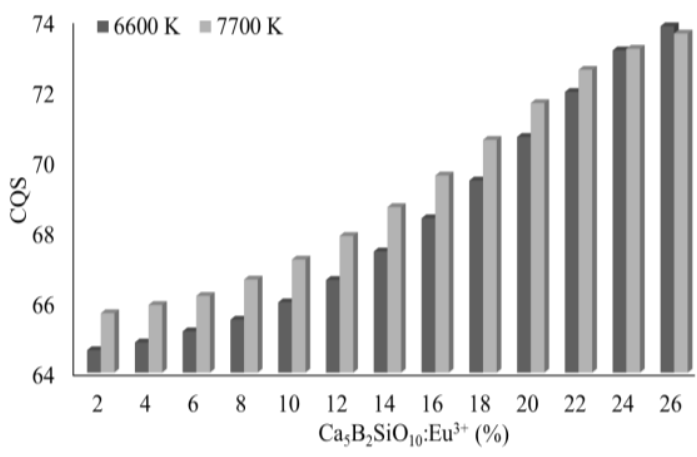

Figure 5. The color quality scale of WLEDs corresponding to $\mathrm{Ca}_{5} \mathrm{~B}_{2} \mathrm{SiO}_{10}: \mathrm{Eu}^{3+}$ concentration

For the typical asymmetrical SPD of monochrome LED modeling process, the Gaussian function is applied [23, 24]:

$$
P_{\lambda}=P_{o p t} \frac{1}{\sqrt{2 \pi}} \exp \left[-0.5^{*} \frac{\left(\lambda-\lambda_{\text {peak }}\right)^{2}}{\sigma^{2}}\right]
$$

Here, $\sigma$ depends on the peak wavelength $\lambda_{\text {peak }}$, and the calculation of FWHM $\Delta \lambda$ can be:

$$
\sigma=\frac{\lambda_{p e a k}^{2} \Delta E}{2 h c \sqrt{2 \operatorname{In} 2}}=\frac{\lambda_{\text {peak }}^{2}\left(\frac{h c}{\lambda_{1}}-\frac{h c}{\lambda_{2}}\right)}{\sqrt[2 h c]{2 \operatorname{In} 2}}=\frac{\lambda_{p e a k}^{2}\left(\frac{h c \Delta \lambda}{\lambda_{1} \lambda_{2}}\right)}{\sqrt[2 h c]{2 \operatorname{In} 2}}
$$

The white-light LED's SPD in connection with the utilized yellow YAG phosphors and blue LED chips can hypothetically be viewed as the aggregate for the spectra of the blue and yellow lights. Practically speaking, nonetheless, the supposed yellow phosphor radiates lights in not only yellow but also green spectra. Given that the blue range and yellow range are selected, it is possible to use the green range to demonstrate the contrast between the essentially estimated SPD and twofold shading (blue and yellow shading) range model. In this way, a green range can be added to the twofold range model, leading to the investigation using trispectrum (B-G-Y) model which is displayed in (3) and after that is replaced altered by the expressions in (4). 


$$
\begin{aligned}
& P_{\lambda}=P_{o p t_{-} b} \frac{1}{\sigma_{b} \sqrt{2 \pi}} \exp \left[-0.5 * \frac{\left(\lambda-\lambda_{p e a k_{-} b}\right)^{2}}{\sigma_{b}^{2}}\right] \\
& +P_{o p t_{-} g} \frac{1}{\sigma_{g} \sqrt{2 \pi}} \exp \left[-0.5 * \frac{\left(\lambda-\lambda_{\text {peak }}\right)^{2}}{\sigma_{g}{ }^{2}}\right] \\
& +P_{o p t_{-} y} \frac{1}{\sigma_{y} \sqrt{2 \pi}} \exp \left[-0.5 * \frac{\left(\lambda-\lambda_{p e a k_{-} y}\right)^{2}}{\sigma_{y}{ }^{2}}\right] \\
& P_{\lambda}=\eta_{b} P_{o p t_{-} \text {total }} \frac{1}{\sigma_{b} \sqrt{2 \pi}} \exp \left[-0.5 * \frac{\left(\lambda-\lambda_{\text {peak_b }_{-}}\right)^{2}}{\sigma_{b}^{2}}\right] \\
& +\eta_{g} P_{\text {opt_total }} \frac{1}{\sigma_{g} \sqrt{2 \pi}} \exp \left[-0.5 * \frac{\left(\lambda-\lambda_{\text {peak_g }}\right)^{2}}{\sigma_{g}{ }^{2}}\right] \\
& +\eta_{y} P_{o p t_{-} \text {total }} \frac{1}{\sigma_{y} \sqrt{2 \pi}} \exp \left[-0.5 * \frac{\left(\lambda-\lambda_{\text {peak }}\right)^{2}}{\sigma_{y}{ }^{2}}\right]
\end{aligned}
$$

In which:

- $\mathrm{P}_{\lambda}$ Spectral power distribution (SPD) ( $\left.\mathrm{mW} / \mathrm{nm}\right)$.

- h Planck's constant (J.s).

- c Speed of light $\left(\mathrm{ms}^{-1}\right)$.

- $\lambda$ Wavelength (nm).

- $\mathrm{P}_{\text {opt }}$ Optical power (W).

- $\lambda_{\text {peak }}$ Peak wavelength (nm).

- $\Delta \lambda$ Full-width at half-maximum (FWHM) (nm).

- $\eta$ Ratio of specific spectra to white spectrum, dimensionless.

- $\mathrm{P}_{\mathrm{opt} \_} \mathrm{b}, \mathrm{P}_{\mathrm{opt} \_\mathrm{g}}, \mathrm{P}_{\mathrm{opt} \_\mathrm{y}}$, and $\mathrm{P}_{\mathrm{opt} \_ \text {total }}$ Optical power $(\mathrm{W})$ for the blue, green, yellow, and white spectra, respectively.

- $\lambda_{\text {peak_b }}, \lambda_{\text {peak_g }}$, and $\lambda_{\text {peak_y }}$ Peak wavelengths $(\mathrm{nm})$ for the blue, green, and yellow spectra, respectively.

- $\sigma_{\mathrm{b}}, \sigma_{\mathrm{g}}$, and $\sigma_{\mathrm{y}}$ FWHM-related coefficients $(\mathrm{nm})$ for the blue, green, and yellow spectra, respectively.

- $\eta_{\mathrm{b}}, \eta_{\mathrm{g}}$, and $\eta_{\mathrm{y}}$ Ratios of blue-green-yellow (B-G-Y) spectra to white spectrum, respectively, dimensionless.

- $\lambda_{1}, \lambda_{2}$ Wavelengths at half of the peak intensity.

Hence, a tricolor spectrum can be used to describe the SPD modeling for the phosphor-coated white LED, and this can be viewed as the extended Gaussian structure. The scattering investigation of $\mathrm{Ca}_{5} \mathrm{~B}_{2} \mathrm{SiO}_{10}: \mathrm{Eu}^{3+}$ particles is conducted with the Mie-scattering theory. Moreover, this theory contributes to computing the scattering cross-section $C_{\text {sca }}$ for spherical particles [25], [26]. Besides that, based on the BeerLambert law, the computation for transmitted light power can be expressed as [27], [28]:

$$
I=I_{0} \exp \left(-\mu_{e x t} L\right)
$$

$I_{0}, \mu_{\mathrm{ext}}$ and $L$ are the incident light power, the extinction coefficient and the thickness of phosphor layer (mm), respectively. Besides, $\mu_{\mathrm{ext}}$ can be expressed as: $\mu_{e x t}=N_{r} . C_{e x t}$, with $N_{r}$ is the number density distribution of particles $\left(\mathrm{mm}^{-3}\right)$, and $C_{\text {ext }}\left(\mathrm{mm}^{2}\right)$ indicates the phosphor particle's extinction cross-section.

By applying expression (5), the outcome shows that dual-layer remote phosphor structure has higher luminous flux than the single-layer phosphor structure. Therefore, the dual-layer phosphor structure actually works better and enables the improvement of luminous flux in WLEDs. Moreover, the calculated results indicated that the concentration of $\mathrm{Ca}_{5} \mathrm{~B}_{2} \mathrm{SiO}_{10}: \mathrm{Eu}^{3+}$ greatly and directly affects the color quality and luminescence performance of WLED dual-layer remote phosphor configuration. In particular, the extinction coefficient $\mu_{\text {ext }}$, demonstrated in the Lambert-Beer law, and the concentration of $\mathrm{Ca}_{5} \mathrm{~B}_{2} \mathrm{SiO}_{10}: \mathrm{Eu}^{3+}$ increase together; however, the light emission energy increase in an inverse direction to the extinction coefficient $\mu_{\text {ext }}$. Consequently, the degradation in lumen output occurs as the phosphor layer is thicken (the concentration of $\mathrm{Ca}_{5} \mathrm{~B}_{2} \mathrm{SiO}_{10}: \mathrm{Eu}^{3+}$ is increased). This point was also described in Figure 6, where the luminous intensity decreases at all CCTs with the rise of $\mathrm{Ca}_{5} \mathrm{~B}_{2} \mathrm{SiO}_{10}: \mathrm{Eu}^{3+}$ concentration, from $0 \%$ to $26 \%$.

Generally, dual-layer phosphor structure with red phosphor $\mathrm{Ca}_{5} \mathrm{~B}_{2} \mathrm{SiO}_{10}: \mathrm{Eu}^{3+}$ already created much better luminous flux than the single-layer. In addition, $\mathrm{Ca}_{5} \mathrm{~B}_{2} \mathrm{SiO}_{10}: \mathrm{Eu}^{3+}$ also gives CRI and CQS many 
benefits. Therefore, a slight reduction in luminous flux is acceptable considering the overall light output and color quality. Thus, the final decision of an appropriate $\mathrm{Ca}_{5} \mathrm{~B}_{2} \mathrm{SiO}_{10}: \mathrm{Eu}^{3+}$ concentration is made by the manufacturers after them considering their goals of WLED products.

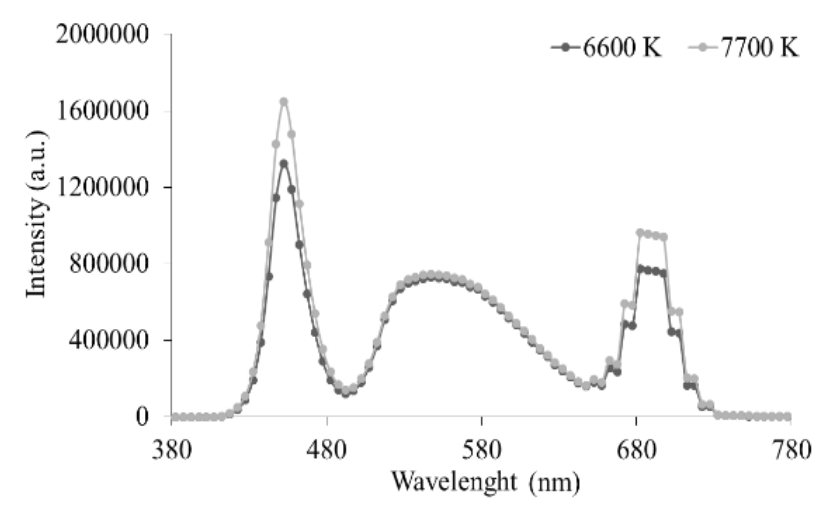

Figure 6. The luminous flux of WLEDs as a function of $\mathrm{Ca}_{5} \mathrm{~B}_{2} \mathrm{SiO}_{10}: \mathrm{Eu}^{3+}$ concentration

\section{CONCLUSION}

This research has successfully applied new technology to create WLEDs with high lighting performance and a changeable emission spectrum. A color design of the WLEDs model is form by applying the Lambert-Beer law and the linear conversion, from which the WLED can adjust its white light to be close to the requirement of manufacturers. The outcomes of the research conducted on the dual-layer phosphor package demonstrate better light output in comparison to the result from the single-layer package. Moreover, the increase in $\mathrm{Ca}_{5} \mathrm{~B}_{2} \mathrm{SiO}_{10}: \mathrm{Eu}^{3+}$ concentration helps the chromatic performance of WLED improve due to the corresponding development of the CRI and CQS. However, the excessive concentration of $\mathrm{Ca}_{5} \mathrm{~B}_{2} \mathrm{SiO}_{10}$ : $\mathrm{Eu}^{3+}$ particles (over 24\%) results in degraded lumen efficiency. With the information from this paper, the concentration of $\mathrm{Ca}_{5} \mathrm{~B}_{2} \mathrm{SiO}_{10}: \mathrm{Eu}^{3+}$ phosphor layer can be determined by manufacturers to address the issues in their production of high-quality WLEDs.

\section{ACKNOWLEDGEMENTS} 21/1.3CB01.

This research is funded by the Industrial University of Ho Chi Minh City under grant number:

\section{REFERENCES}

[1] H. Yang, Lei Zhang, Y. Yuan, W. Xiang, Y. Cui and J. Zhang, "“Giant" quantum dots encapsulated inside a freeform lens," Applied Optics, vol. 57, no. 35, pp. 10317-10322, 2018, doi: 10.1364/AO.57.010317.

[2] G. Prabhakar, P. Gregg, L. Rishoj, P. Kristensen, and S. Ramachandran, "Octave-wide supercontinuum generation of light-carrying orbital angular momentum," Optics Express, vol. 27, no. 8, pp. 11547-11556, 2019, doi: 10.1364/OE.27.011547.

[3] Y. F. Huang, Yu-Chieh Chi, Mu-Ku Chen, Din Ping Tsai, Ding-Wei Huang and Gong-Ru Lin, "Red/green/blue LD mixed white-light communication at $6500 \mathrm{~K}$ with divergent diffuser optimization," Optics Express, vol. 26, no. 18, pp. 23397-23410, 2018, doi: 10.1364/OE.26.023397.

[4] L. Yang, Qin Zhang, Feng Li, An Xie, Long Mao and Jidong Ma, "Thermally stable lead-free phosphor in glass enhancement performance of light emitting diodes application," Applied Optics, vol. 58, no. 15, pp. 4099-4104, 2019, doi: 10.1364/AO.58.004099.

[5] McDonnell, E. Coyne, and G. M. O'Connor, "Grey-scale silicon diffractive optics for selective laser ablation of thin conductive films," Applied Optics, vol. 57, no. 24, pp. 6966-6970, 2018, doi: 10.1364/AO.57.006966.

[6] X. Yang, Chufen Chai, Jiachao Chen, Songsheng Zheng, and Chao Chen, "Single $395 \mathrm{~nm}$ excitation warm WLED with a luminous efficiency of $104.86 \mathrm{~lm} / \mathrm{W}$ and a color rendering index of 90.7," Optical Materials Express, vol. 9 , no. 11, pp. 4273-4286, 2019, doi: 10.1364/OME.9.004273.

[7] Swiatczak, Marita Feldkaemper, and Frank Schaeffel, "Changes in fundus reflectivity during myopia development in chickens," Biomedical Optics Express, vol. 10, no. 4, pp. 1822-1840, 2019, doi: 10.1364/BOE.10.001822.

[8] H. Chen, X. Z. Lai, P. Chen, Y. T. Liu, M. Y. Yu, Z. H. Liu et al., "Quadrichromatic LED based mobile phone camera visible light communication," Optics Express, vol. 26, no. 13. pp. 17132-17144, 2018, doi: 10.1364/OE.26.017132. 
[9] S. P. Groth, Athanasios G. Polimeridis, Alexandra Tambova, and Jacob K. White, "Circulant preconditioning in the volume integral equation method for silicon photonics," Journal of the Optical Society of America A, vol. 36, no. 6. pp. 1079-1088, 2019, doi: 10.1364/JOSAA.36.001079

[10] J. Zhang, Lei Zhao, Xintian Bian, and Guibin Chen, "Ce3+/Mn2+-activated Ca7, PO4.2, SiO4.2: efficient luminescent materials for multifunctional applications," Optics Express, vol. 26, no. 18, pp. A904-A914, 2018, doi: 10.1364/OE.26.00A904.

[11] R. Wan, Shuo Zhang, Zhiqiang Liu and Xiaoyan Yi, "Simultaneously improve the luminous efficiency and colorrendering index of GaN-based white-light-emitting diodes using metal localized surface plasmon resonance," Optics Letters, vol. 44, no. 17, pp. 4155-4158, 2019, doi: 10.1364/OL.44.004155.

[12] H. L. Ke, Jian Hao, Jian-Hui Tu, Miao Peixian, "Lumen degradation analysis of LED lamps based on the subsystem isolation method," Applied Optics, vol. 57, no. 4, pp. 849-854, 2018, doi: 10.1364/AO.57.000849.

[13] C. Zhang, H. Zhang, R. Wang, D. You, W. Wang, C. Xu et al., "Exciton photoluminescence of CsPbBr $@ \mathrm{SiO}_{2}$ quantum dots and its application as a phosphor material in light-emitting devices," Optical Materials Express, vol. 10, no. 4, pp. 1007-1017, 2020, doi: 10.1364/OME.389847.

[14] N. Rashid, N. H. Ngajikin, A. I. Azmi, R. Arsat, S. Isaak, N. A. Cholan et al., "Spectrophotometer with enhanced sensitivity for uric acid detection," Chinese Optics Letters, vol. 17, no. 8, 2019, Art. no. 081701.

[15] S. Beldi, F. Boussaha, J. Hu, A. Monfardini, A. Traini, F. Levy-Bertrand et al., "High Q-factor near infrared and visible Al2O3-based parallel-plate capacitor kinetic inductance detectors," Optics Express, vol. 27, no. 9, pp. 13319-13328, 2019, doi: 10.1364/OE.27.013319.

[16] J. Hao, Hong-Liang Ke, Lei Jing, Qiang Sun, "Prediction of lifetime by lumen degradation and color shift for LED lamps, in a non-accelerated reliability test over 20,000 h," Applied Optics, vol. 58, no. 7, pp. 1855-1861, 2019, doi: 10.1364/AO.58.001855

[17] X. Li, B. Hussain, L. Wang, J. Jiang and C. P. Yue, "Design of a 2.2-mW 24-Mb/s CMOS VLC Receiver SoC With Ambient Light Rejection and Post-Equalization for Li-Fi Applications," Journal of Lightwave Technology, vol. 36, no. 12, pp. 2366-2375, 2018, doi: 10.1109/JLT.2018.2813302.

[18] Q. Zhang, Ruilin Zheng, Jianyong Ding, and Wei Wei, "Excellent luminous efficiency and high thermal stability of glass-in-LuAG ceramic for laser-diode-pumped green-emitting phosphor," Optics Letters, vol. 43, no. 15, pp. 3566-3569, 2018, doi: 10.1364/OL.43.003566.

[19] X. Liu, R. Lei, F. Huang, D. Deng, H. Wang, S. Zhao et al., "Upconversion luminescence, intrinsic optical bistability, and optical thermometry in Ho3+/Yb3+: BaMoO4 phosphors," Chinese Optics Letters, vol. 17, no. 11, pp. 111601, 2019.

[20] Q. Zaman, J. Souza, O. Pandoli, K. Q. Costa, V. Dmitriev, D. Fulvio et al., "Two-color surface plasmon resonance nanosizer for gold nanoparticles," Optics Express, vol. 27, no. 3, pp. 3200-3216, 2019, doi: 10.1364/OE.27.003200.

[21] M. Dupont-Nivet, I. Westbrook, and S. Schwartz, "The role of trap symmetry in an atom-chip interferometer above the Bose-Einstein condensation threshold," in 2019 Conference on Lasers and Electro-Optics Europe and European Quantum Electronics Conference, OSA Technical Digest, Optical Society of America, 2019.

[22] X. Hu, Y. Lian, Z. Liu, Y. Jin, Y. Hu, Y. Liu et al., "Optimizing selection of the test color sample set for the CIE 2017 color fidelity index," Optics Express, vol. 28, no. 6, pp. 8407-8422, 2020, doi: 10.1364/OE.383283.

[23] M. M. Magno-Canto, Lachlan I. W. McKinna, Barbara J. Robson, and Katharina E. Fabricius, "Model for deriving benthic irradiance in the Great Barrier Reef from MODIS satellite imagery," Optics Express, vol. 27, no. 20, pp. A1350-A1371, 2019, doi: 10.1364/OE.27.0A1350.

[24] Q. T. Vinh, Peter Bodrogi, and Tran Quoc Khanh, "Preliminary measure for the characterization of the usefulness of light sources," Optics Express, vol. 26, no. 11, pp. 14538-14551, 2018, doi: 10.1364/OE.26.014538.

[25] H. Gu, Mengzhu Chen, Qixia Wang, and Qiaofeng Tan, "Design of two-dimensional diffractive optical elements for beam shaping of multicolor light-emitting diodes," Applied Optics, vol. 57, no. 10, pp. 2653-2658, 2018, doi: 10.1364/AO.57.002653.

[26] M. K. Kang, L. Sisken, J. Cook, C. Blanco, M. C. Richardson, I. Mingareev et al., "Refractive index patterning of infrared glass ceramics through laser-induced vitrification [Invited]," Optical Materials Express, vol. 8, no. 9, pp. 2722-2733, 2018, doi: 10.1364/OME.8.002722

[27] Q. Song, S. Chen, C. Xue, M. Lin, K. Du, S. Li et al., "Vicarious calibration of COCTS-HY1C at visible and nearinfrared bands for ocean color application," Optics Express, vol. 27, no. 20, pp. A1615-A1626, 2019, doi: 10.1364/OE.27.0A1615.

[28] P. P. Li, Y. B. Zhao, H. J. Li, J. M. Che, Z. H. Zhang, Z. C. Li et al., "Very high external quantum efficiency and wall-plug efficiency $527 \mathrm{~nm}$ InGaN green LEDs by MOCVD," Optics Express, vol. 26, no. 25, pp. 33108-33115, 2018, doi: 10.1364/OE.26.033108. 